\title{
Twelve-month follow-up after posterior 25G vitrectomy due to idiopathic full-thickness macular hole treated with "inverted ILM flap" technique
}

\author{
Michał Patyk', Mariusz Kosatka', Adam Kluś1', Janusz Sierdziński', Marek Rękas' \\ 'Department of Ophthalmology, Military Institute of Medicine in Warsaw, Poland \\ ${ }^{2}$ Department of Medical Informatics and Telemedicine of the Medical University of Warsaw, Poland
}

\begin{abstract}
BACKGROUND: We conducted a prospective, follow-up study to analyze a long-term recovery process after posterior vitrectomy for idiopathic full-thickness macular hole.

MATERIAL AND METHODS: Sixty eyes were evaluated in 59 patients who underwent surgery for idiopathic fullthickness macular hole using posterior $25 \mathrm{G}$ vitrectomy with the "inverted ILM flap" technique from the beginning of 2013 to the end of 2014. In 55 eyes, posterior 25G phaco-vitrectomy with ILM peeling was performed using the "inverted ILM flap" technique with IOL implantation. In 5 pseudophakic eyes, posterior vitrectomy was performed with the technique as above. All patients were interviewed regarding the duration of the disease and pre- and postoperatively at intervals of $1,3,6$, and 12 months. The following tests were performed: best-corrected visual acuity (BCVA), best-corrected near visual acuity (BCNVA), intraocular pressure (IOP) measurement, physical examination of the anterior and posterior segment of the eye, and spectral domain optical coherence tomography (SD-OCT). RESULTS: The anatomical success in the study was $95 \%$, and we achieved the improvement in visual acuity in $91.7 \%$ of the operated eyes. The visual acuity in the time interval before surgery and 12 months afterward improved from 0.1 to 0.29 and was statistically significant $(\mathrm{p}<0.05)$. Shorter duration and smaller hole size were significant predictors of improved postoperative visual acuity. The U-type of the hole closure predisposed to the most significant improvement in visual acuity in patients.

CONCLUSIONS: The obtained functional and anatomical results may confirm the effectiveness of posterior vitrectomy using the "inverted ILM flap" technique in treating idiopathic full-thickness macular holes. The observed sparce complications did not differ from the complication profile described by other authors.
\end{abstract}

KEY WORDS: idiopathic full-thickness macular hole; posterior vitrectomy; inverted ILM flap technique

Ophthalmol J 2021; Vol. 6, 274-280

CORRESPONDING AUTHOR:

Michał Patyk, MD, Department of Ophthalmology, Military Institute of Medicine in Warsaw, 128 Szaserow Str., Warsaw, Poland; e-mail: micpat@wp.pl 


\section{INTRODUCTION}

An idiopathic full-thickness macular hole is a disease that commonly occurs in individuals aged 60-70 years, and women are affected more frequently. For over a dozen years now, owing to the discoveries of Kelly and Wendel, there has been a possibility of effective surgical treatment of this disease [1]. As a result of the constant modernization of operating devices in optics, surgical instruments, and surgeons' skills, anatomical success reaches over $90 \%$ in many centers and the functional success around $80 \%[2,3]$.

The postoperative outcomes of full-thickness macular hole closure, especially in the eyes accompanied by high myopia and large holes, were significantly improved by the "inverted ILM flap" technique introduced and described by prof. Jerzy Nawrocki in 2010 [4]. The surgeries of full-thickness macular holes with the use of this surgical technique have been performed at the Department of Ophthalmology of the Military Medical Institute in Warsaw, Poland, since 2012.

The aim of the study was to evaluate the efficacy and safety of surgical treatment of idiopathic full-thickness macular holes using posterior 25G vitrectomy with the "inverted ILM flap" technique.

\section{MATERIAL AND METHODS}

The study included 60 eyes in 59 patients with idiopathic full-thickness macular holes (FTMH), qualified and operated at the Department of Ophthalmology of the Military Institute of Medicine in Warsaw from January 1, 2013, to December 31, 2014.

Patients with coexisting FTMH retinal detachment, post-traumatic FTMH, with concomitant high myopia (greater than $-6 \mathrm{D}$ ), after previous vitreoretinal surgeries, with advanced glaucoma, previous uveitis, history of endophthalmitis, advanced diabetic retinopathy, and advanced age-related macular degeneration (AMD) lesions, were excluded from the study.

Before the surgery, each patient was interviewed regarding the duration of disease symptoms. The best-corrected visual acuity (BCVA) was performed using Snellen charts. The intraocular pressure was measured using the applanation method (Goldmann applanation tonometer). Anterior and posterior eye segments were examined (Zeiss SL 115), and optical coherence tomography (OCT SLO OTI) was performed.
Forty-nine women and ten men were qualified for surgery. The mean age of the patients was 69.2 years ( $48-84$ years).

All patients underwent posterior vitrectomy with the use of the inverted internal limiting membrane flap technique (ILM). Two vitreoretinal surgeons performed the surgery. All posterior vitrectomy procedures were conducted with $25 \mathrm{G}$ instruments. Retrobulbar anesthesia was used. Four trocars were inserted into the vitreous chamber through the flat part of the ciliary body $3.5 \mathrm{~mm}$ from the limbus in four quadrants (Fig. 1A). This was followed by a central (Fig. 1B) and peripheral (Fig. 1C) vitrectomy. Membrane Blue Dual was used during a posterior vitrectomy for ILM staining (Fig. 1D). ILM peeling was performed using Eckardt forceps and was conducted circularly around the hole to the size of approximately 2 disc diameters. ILM was not completely removed, but its central part next to the hole was left and then trimmed and manipulated over the macular hole (Fig. 1E), with a gentle massage with forceps such that the ILM flap remained inverted in the hole. The final stage of the surgery was a fluid-air exchange, followed by the injection of 20\% SF6 gas into the vitreous chamber (Fig. $1 \mathrm{~F}$ ) and sealing of the ports after sclerotomies.

All patients were advised "face down" position for three days after surgery ( $90 \%$ of the time during the day).

\section{RESULTS \\ Evaluation of the obtained functional and anatomical results}

One month after the surgery, the macular hole closure was achieved in 57 cases (95\%). In 3 cases $(5 \%)$, the macular hole was not closed. The reopening of closed macular holes was not reported in the 12-month follow-up.

The functional success achieved in the study was $91.7 \%$. BCVA before surgery was $0.11 \pm 0.05$ for the study group of patients; the postoperative BCVA value after 12 -month follow-up was $0.29 \pm 0.14$. The increase in BCVA in each time interval was statistically significant $(\mathrm{p}<0.001)$ (Tab. 1). A gain of 1 or more lines on the Snellen chart was seen in 54 patients; most often - in 16 patients - the gain was two lines (Fig. 2).

The study measured the effect on final visual acuity at 12 months: disease duration, macular hole size, and type of macular hole closure. 


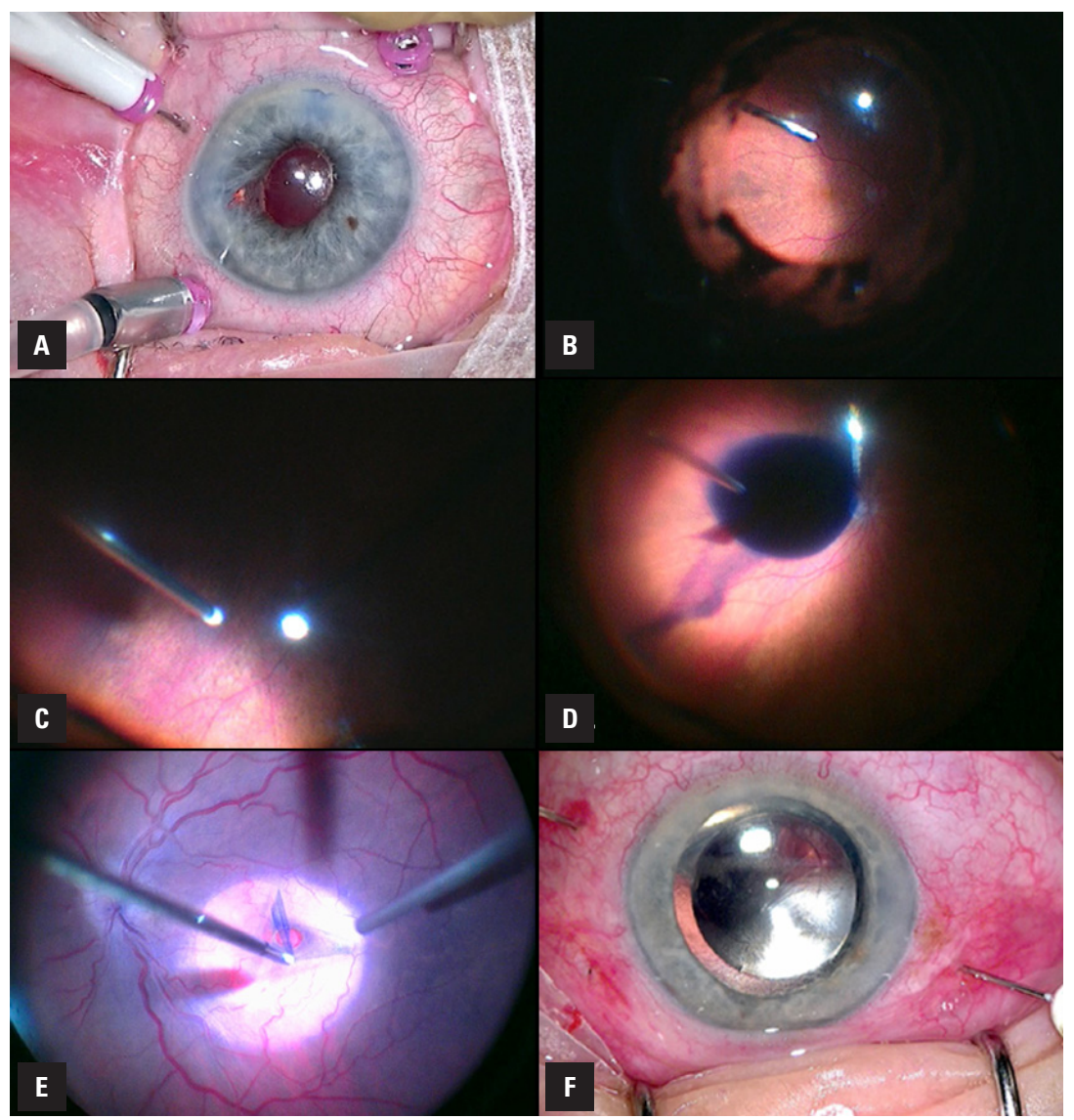

FIGURE 1. Stages of posterior 25G vitrectomy for the treatment of full-thickness macular holes using the "inverted ILM flap" method (material from the surgeries performed in the study)

Table 1. Best-corrected visual acuity (BCVA) at consecutive 12-month follow-up intervals; Friedman's ANOVA showed significant statistical differences for the BCVA at all time points $\mathrm{Ch}^{-2}=163.36$ for $\mathrm{p}<0.001$

\begin{tabular}{|l|c|c|c|c|c|}
\hline Follow-up & $\begin{array}{c}\mathbf{0} \\
\text { Pre op }\end{array}$ & $\mathbf{1}$ month & $\mathbf{3}$ months & $\mathbf{6}$ months & 12 months \\
\hline BCVA & $0.11 \pm 0.05$ & $0.17 \pm 0.11$ & $0.24 \pm 0.12$ & $0.27 \pm 0.13$ & $0.29 \pm 0.14$ \\
\hline p-value & & $<0.001$ & $<0.001$ & $<0.001$ & $<0.001$ \\
\hline
\end{tabular}

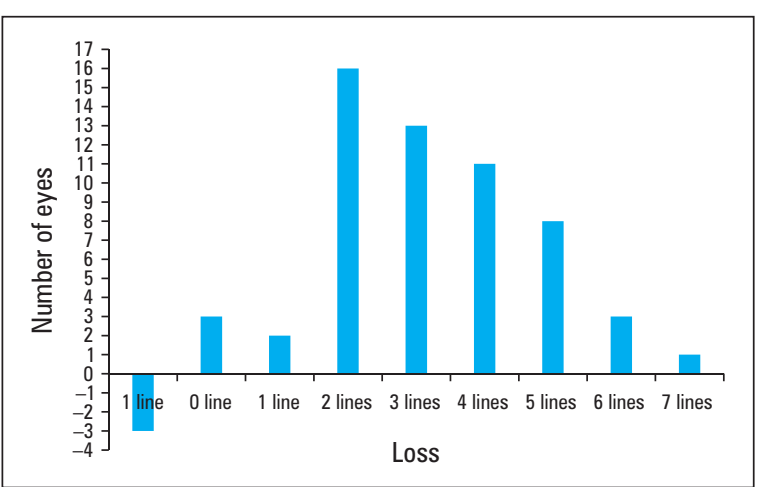

FIGURE 2. Snellen line gain/loss after 12-month follow-up

\section{Effect of disease symptoms duration on postoperative visual acuity}

The correlation between BCVA at the end of the 12-month follow-up on the macular hole duration $(\mathrm{T})$ is described by the regression equation:

$$
B C V A=0.423-0,0083 * T .
$$

The shorter duration of disease symptoms predisposed to better BCVA at the end of follow-up (Fig. 3).

Effect of macular hole size on postoperative visual acuity 


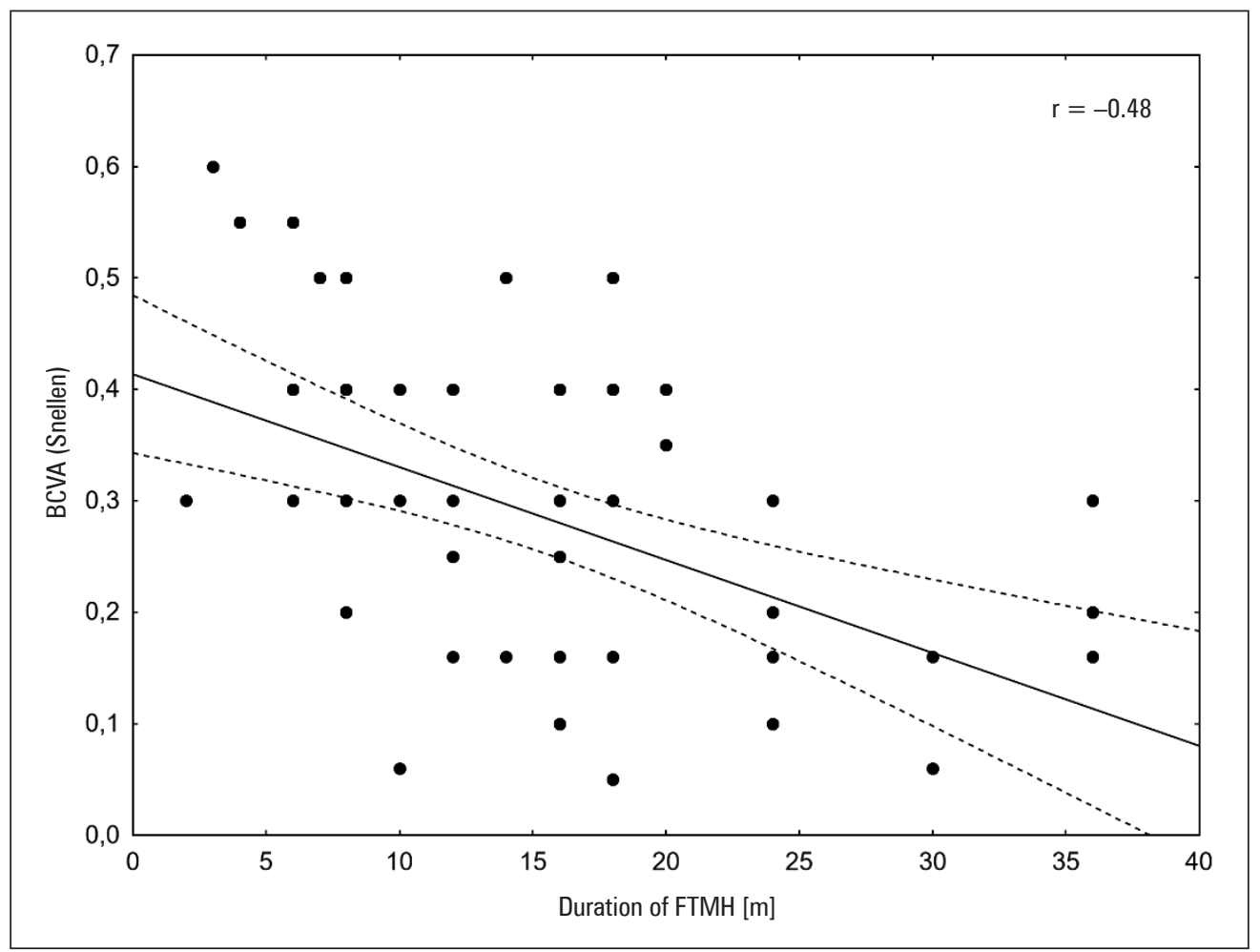

FIGURE 3. Correlation between best-corrected visual acuity (BCVA) and the duration of the macular hole(months)

The study revealed a strong correlation between the smallest macular hole size (Wo_MIN) and the hole size measured at the base (Wo_basal) (Fig. 4). Further analyzes were performed only for Wo_MIN.

The correlation between the best visual acuity (BCVA) at the end of postoperative follow-up and the size of the hole (Wo_MIN) is described by the regression equation:

$$
B C V A=0.5804-0.0005 * \text { Wo_MIN }
$$

The smaller hole size (Wo_MIN) predisposed to better visual acuity at the end of the follow-up (Fig. 5).

\section{Effect of the macular hole closure type on postoperative visual acuity}

In 21 cases, the surgery resulted in a U-type closure (35\% of cases), in 11 cases, a V-type closure ( $18.3 \%$ of cases), and in 25 cases, a W-type closure ( $41.7 \%$ of cases).

In 3 cases, the macular hole was not closed (5\% of cases) (Fig. 6).

In the U-type closure group there was a significant improvement in BCVA from $0.13 \pm 0.05$ to $0.4 \pm 0.17(\mathrm{p}<0.001)$, in the $\mathrm{V}$-type closure group BCVA improved from $0.09 \pm 0.05$ to $0.29 \pm 0.12(\mathrm{p}<0.001)$, and in the W-type closure group from $0.1 \pm 0.05$ to $0.23 \pm 0,1(\mathrm{p}<0.001)$ after 12 month follow-up. If the hole was not closed after 12 month follow-up, the BCVA deteriorated from $0.09 \pm 0.02$ to $0.07 \pm 0.03)(\mathrm{p}=0.663)$ (Tab. 2).

In the case of $U_{-}, W_{-}$, and V-type closure, there was a significant improvement in BCVA 1 month after surgery $(p=0.001, p=0.005, p=0.02)$, and visual acuity improved by the end of the follow-up (Fig. 8).

\section{Complications}

At each postoperative follow-up interval, no patients experienced significant increases in IOP. There were no serious complications such as hemorrhage into the vitreous chamber, retinal tears, and detachment or endophthalmitis in any of the patients.

\section{DISCUSSION}

The development of surgical instruments, especially the appropriate technique and surgical experience, increased the effectiveness of treatment in the case of idiopathic full-thickness macular holes. After introducing posterior vitrectomy as a method of macular hole treatment, the effectiveness was initially about $40-50 \%[2,3]$. Owing to the im- 


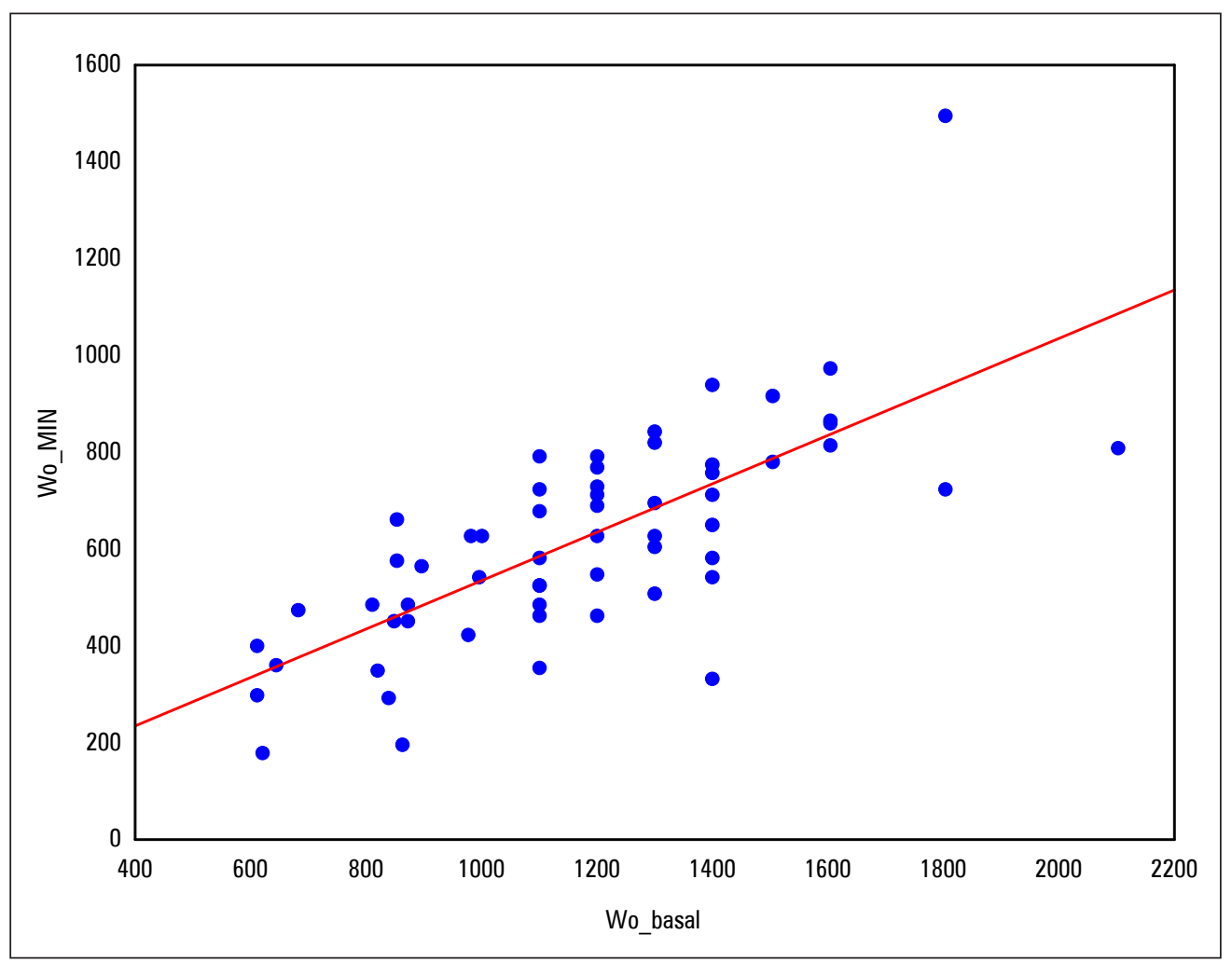

FIGURE 4. Correlation between the minimum size of the macular hole and that measured at the base.

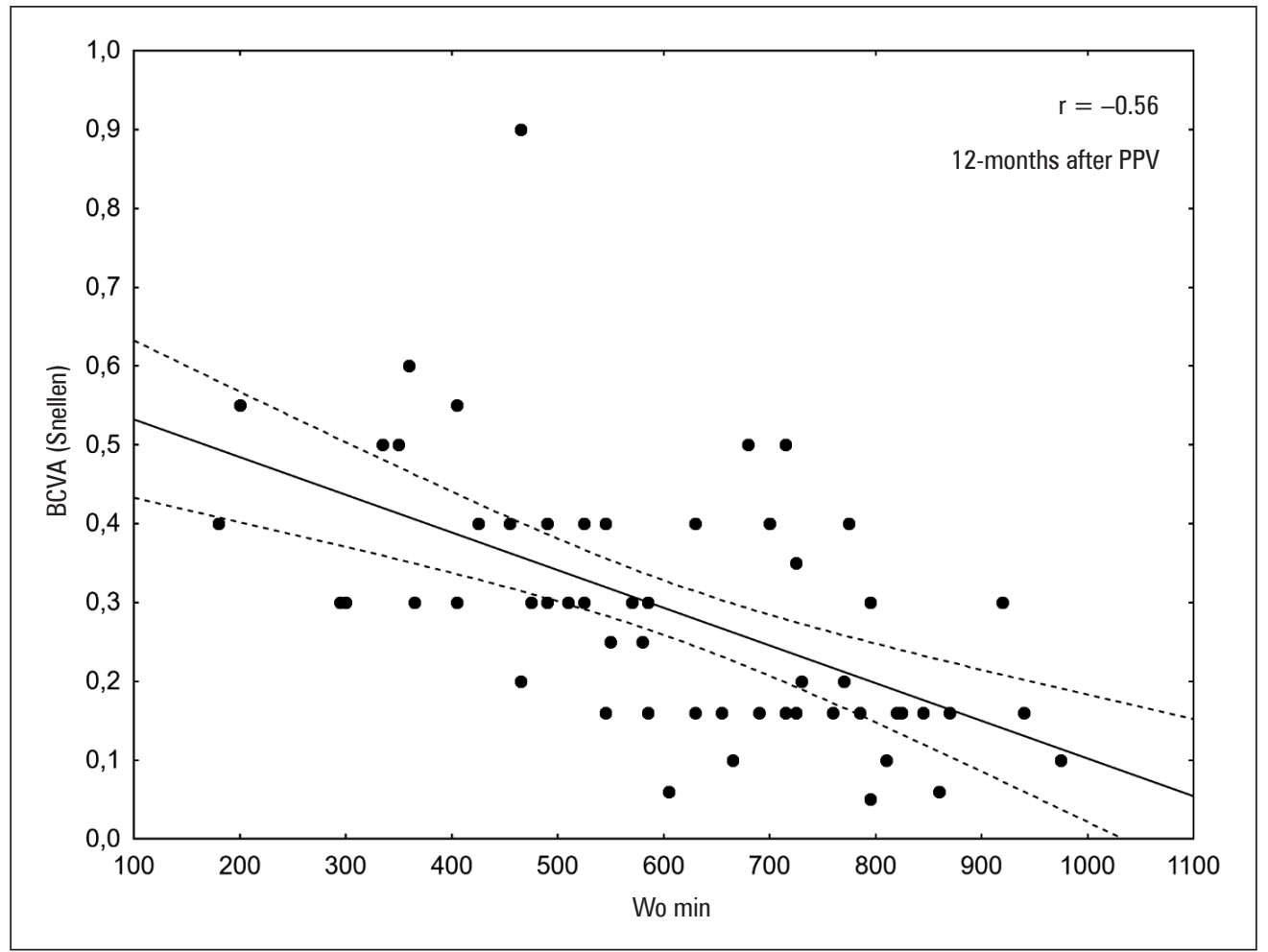

FIGURE 5. Correlation between best-correct visual acuity (BCVA) and the smallest macular hole size (Wo_MIN) 


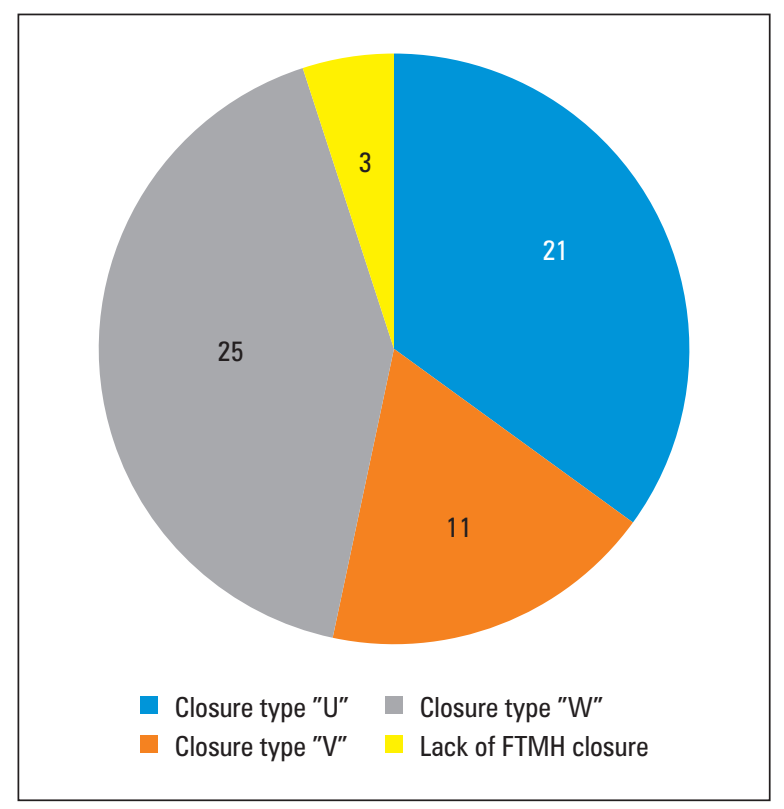

FIGURE 6. The distribution of different types of macular hole closure after 12-month follow-up

provement of the technique, the surgical success, understood as the closure of the macular hole, is often over $90 \%$ after the first surgery [4-6], and the functional success is over $80 \%$. However, there is still a problem with full-thickness macular holes in patients with large macular holes and high myopia $[2,3,7]$. Introduction of the "inverted ILM flap" technique by prof. Nawrocki resulted in much greater effectiveness of treatment in patients with high myopia and with large macular holes $[4,8]$. It is also, very importantly, a surgery that can be repeated in the same patient after the failure of macular hole closure, e.g., in the case of ILM flap instability. In this study, the macular hole was closed in $95 \%$, and visual acuity improved in $91.7 \%$ of cases. These results are comparable with previous studies $[4,8]$. No case of macular hole reopening was observed during postoperative follow-up.

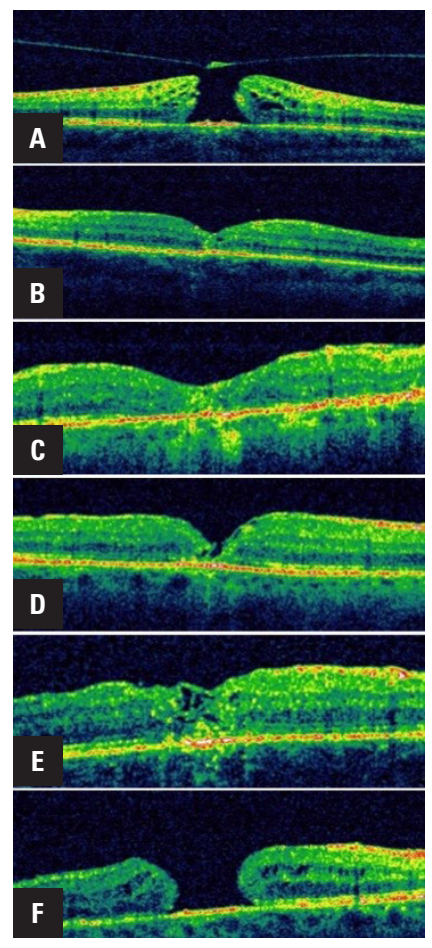

FIGURE 7. Optical coherence tomography (OCT). A. Full-thickness macular hole; B.-E. Condition after full-thickness macular hole closure; B., C. U-type closure, the closest to the physiological; D. V-type closure, with steep edges; E. W-type closure, irregular; F. Lack of macular hole closure (material from the surgeries performed in the study)

The study proved that a shorter duration of symptoms was associated with better postoperative visual acuity. The shorter the opening time until surgery - up to about 12 months - the smaller the macular hole was, and after the surgery, a U-type closure was observed, which predisposed to better visual acuity (Fig. 8, Tab.2). In patients with a much longer medical history - over 18 months (Fig. 3, Tab. 2), macular holes were most often larger than $700 \mu \mathrm{m}$, and after the surgery, the W-type closure was observed — irregular, which had a significantly worse prognosis.

Table 2. Distance best-corrected visual acuity (BCVA) depending on the type of macular hole closure at each follow-up
period
\begin{tabular}{|l|c|c|c|c|c|}
\hline $\begin{array}{l}\text { Time } \\
\text { Type of closure }\end{array}$ & Pre-op & 1 month & 3 months & 6 months & 12 months \\
\hline U & $0.13 \pm 0.05$ & $0.24 \pm 0.13$ & $0.32 \pm 0.12$ & $0.37 \pm 0.14$ & $0.4 \pm 0.17$ \\
\hline V & $0.09 \pm 0.05$ & $0.14 \pm 0.08$ & $0.21 \pm 0.1$ & $0.24 \pm 0.1$ & $0.29 \pm 0.12$ \\
\hline W & $0.1 \pm 0.05$ & $0.13 \pm 0.06$ & $0.2 \pm 0.09$ & $0.22 \pm 0.1$ & $0.23 \pm 0.1$ \\
\hline Lack of closure & $0.09 \pm 0.02$ & $0.09 \pm 0.02$ & $0.1 \pm 0.05$ & $0.07 \pm 0.03$ & $0.07 \pm 0.03$ \\
\hline p & 0.08 & 0.0082 & 0.0008 & 0.0002 & 0.0001 \\
\hline
\end{tabular}




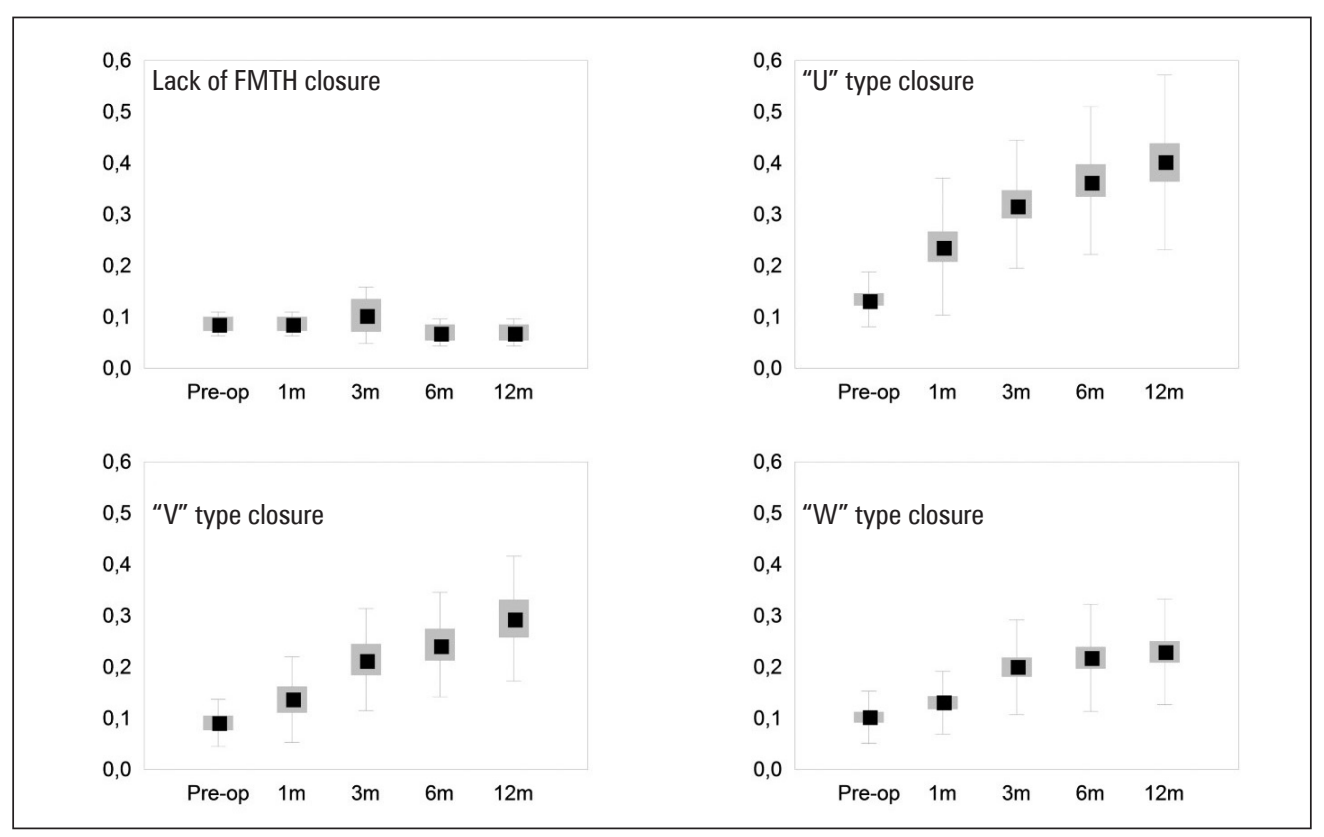

FIGURE 8. Correlation between best-corrected visual acuity (BCVA) and the type of macular hole closure before surgery and in subsequent postoperative follow-up intervals

A smaller macular hole size predisposed to better postoperative visual acuity in the conducted study. The best results were achieved in patients with a macular hole size of less than $400 \mu \mathrm{m}$, and significantly worse outcomes in those with a macular hole size greater than $800 \mu \mathrm{m}$. This is consistent with the observations of Ulrich et al., who in their study showed a correlation between the macular hole size and the functional result after the macular hole closure [9]. The best functional results were achieved in patients with postoperative U-type macular hole closure. Similar conclusions were presented in the study of Kang [10] and Michalewska [4].

\section{CONCLUSION}

It should be stated that posterior $25 \mathrm{G}$ vitrectomy using the "inverted ILM flap" technique is an effective and safe method of idiopathic macular hole treatment.

\section{REFERENCES}

1. Kelly NE, Wendel RT. Vitreous Surgery for Idiopathic Macular Holes: Results of a Pilot Study. Retina. 1991; 11(4): 447, doi: 10.1097/00006982199111040-00026, indexed in Pubmed: 2025167.

2. Ezra E, Gregor ZJ. Morfields Macular Hole Study Ggroup Report No. 1. Surgery for idiopathic full-thickness macular hole: two-year results of a randomized clinical trial comparing natural history, vitrectomy, and vitrectomy plus autologous serum: Morfields Macular Hole Study Group RAeport no. 1. Arch Ophthalmol. 2004; 122(2): 224-236, doi: 10.1001/archopht.122.2.224, indexed in Pubmed: 14769600.

3. Ip MS, Baker BJ, Duker JS, et al. Anatomical outcomes of surgery for idiopathic macular hole as determined by optical coherence tomography. Arch Ophthalmol. 2002; 120(1): 29-35, doi: 10.1001/ archopht.120.1.29, indexed in Pubmed: 11786054.

4. Michalewska Z, Michalewski J, Adelman RA, et al. Inverted internal limiting membrane flap technique for large macular holes. Ophthalmology. 2010; 117(10): 2018-2025, doi: 10.1016/j.ophtha.2010.02.011, indexed in Pubmed: 20541263.

5. Ando F, Sasano K, Ohba N, et al. Anatomic and visual outcomes after indocyanine green-assisted peeling of the retinal internal limiting membrane in idiopathic macular hole surgery. Am J Ophthalmol. 2004; 137(4): 609-614, doi: 10.1016/j.ajo.2003.08.038, indexed in Pubmed: 15059697.

6. Beutel J, Dahmen G, Ziegler A, et al. Internal limiting membrane peeling with indocyanine green or trypan blue in macular hole surgery: a randomized trial. Arch Ophthalmol. 2007; 125(3): 326-332, doi: 10.1001/ archopht.125.3.326, indexed in Pubmed: 17353402.

7. Caicedo A, Espinosa-Heidmann DG, Piña Y, et al. Blood-derived macrophages infiltrate the retina and activate Muller glial cells under experimental choroidal neovascularization. Exp Eye Res. 2005; 81(1): 38-47, doi: 10.1016/j.exer.2005.01.013, indexed in Pubmed: 15978253.

8. Mete $M$, Alfano A, Guerriero $M$, et al. Inverted internal limiting mambrane flap technique versus complete internal limiting membrane removal in myopic macular hole surgery: A Comparative Study. Retina. 2017; 37(10): 1923-1930, doi: 10.1097/IAE.0000000000001446, indexed in Pubmed: 28067723.

9. Ullrich S, Haritoglou C, Gass C, et al. Macular hole size as a prognostic factor in macular hole surgery. Br J Ophthalmol. 2002; 86(4): 390-393, doi: 10.1136/bjo.86.4.390, indexed in Pubmed: 11914205.

10. Kang SW, Ahn K, Ham Dl. Types of macular hole closure and their clinical implications. Br J Ophthalmol. 2003; 87(8): 1015-1019, doi: 10.1136/bjo.87.8.1015, indexed in Pubmed: 12881347. 